\title{
The Epidemiology of Cystic Fibrosis in Arab Countries: A Systematic Review
}

\author{
Samer Hammoudeh ${ }^{1} \cdot$ Wessam Gadelhaq $^{2} \cdot$ Yahya Hani $^{1} \cdot$ Nadia Omar $^{1} \cdot$ Darine El Dimassi $^{1} \cdot$ Cynthia Elizabeth $^{1}$. \\ Abdul Kareem Pullattayil ${ }^{3}$. Prem Chandra ${ }^{1} \cdot$ Ibrahim A. Janahi ${ }^{2,4}$
}

Accepted: 11 January 2021 / Published online: 30 January 2021

(C) The Author(s) 2021

\begin{abstract}
Cystic fibrosis (CF) is a genetic autosomal recessive disease that involves multiple systems. Both life quality and expectation are affected by the debilitating multi-system involvement of the disease which includes pulmonary, gastrointestinal, reproductive, and endocrine symptoms. Very few studies have covered the epidemiological aspects of the disease among Arab countries. To systematically review the available epidemiological literature on cystic fibrosis in order to describe the epidemiological state of this disease in the Arab world, this review used PubMed to search for relevant articles related to patients with cystic fibrosis, with no restriction on gender or age. Google scholar and the snowballing technique were used to locate further articles. A total of 17 articles met the inclusion criteria for this review. These articles were from Bahrain, Egypt, Jordan, Kuwait, Morocco, Oman, Saudi Arabia, Tunisia, and the United Arab Emirates. The articles scored 1.5-6.5 out of 8 on the quality assessment. The epidemiological data reported varied based on country and type of study. The establishment of CF registries is definitely a priority in the region, as well as the need for more research involving CF patients particularly those involving the epidemiological and clinical characteristics of these patients.
\end{abstract}

Keywords Arab $\cdot$ Middle East $\cdot$ Qatar $\cdot$ Cystic fibrosis $\cdot$ Systematic review

$\begin{array}{ll}\text { Abbreviations } \\ \text { CF } & \text { Cystic fibrosis } \\ \text { CFTR } & \begin{array}{l}\text { Cystic fibrosis transmembrane } \\ \text { conductance regulator }\end{array} \\ \text { OMC } & \begin{array}{l}\text { Oman Medical College } \\ \text { PRISMA }\end{array} \\ & \begin{array}{l}\text { Preferred Reporting Items for } \\ \text { Systematic Reviews and Meta-Analyses }\end{array} \\ \text { UAE } & \text { United Arab Emirates }\end{array}$

This article is part of the Topical Collection on Medicine

Ibrahim A. Janahi ijanahi@sidra.org

1 Medical Research Center, Research Affairs, Hamad Medical Corporation, PO Box 3050, Doha, Qatar

2 Medical Education, Sidra Medicine, PO Box 26999, Doha, Qatar

3 Library Services, Sidra Medicine, PO Box 26999, Doha, Qatar

4 Pediatric Pulmonology, Pediatric Medicine, Sidra Medicine, PO Box 26999, Doha, Qatar

\section{Introduction}

Cystic fibrosis (CF) is a genetic autosomal recessive disease that involves multiple systems [1] and affects more than 72,000 patients globally [2]. The prevalence ranges from 1 in $2000-100,000$ based on country [3]. In the Middle East, the prevalence is estimated at 1 in $30,000-50,000$, while the incidence is estimated at 1 in 2000-5800 live births [4].

Both life quality and expectation are affected by the debilitating multi-system involvement of the disease which includes pulmonary, gastrointestinal, reproductive and endocrine symptoms [5]. The median age of survival is 10-20 years in Arab countries [4] compared to 40 years and more in developed countries [1]. The pathophysiology of CF involves a mutation in the gene that encodes the CF transmembrane conductance regulator (CFTR) protein [4], with the number of mutations exceeding 2000 so far [1]. These mutations are categorized into six categories based on the defect in the CFTR [6].

Current studies involving cystic fibrosis in Arab countries cover a range of topics including epidemiology, clinical manifestations, and mutations. Very few studies have covered the epidemiological aspects of the disease among Arab countries, 
and these also vary in their methodology as well as sample size. This paper aims to expand on previous work by gathering estimates of $\mathrm{CF}$ patients in the Arab world, which in turn would aid in determining the size and capacity of future local and regional efforts, as well as an attempt to lay the foundation for future necessary work involving CF in Arab countries.

\section{Methods}

\section{Electronic databases}

We searched PubMed (Feb 4, 2019) for relevant articles related to patients with $\mathrm{CF}$, with no restriction on gender or age. Google scholar was used to search for further literature. In addition, the snowballing technique [7] was used to locate further articles. A second search (Sep 22, 2019) was conducted in order to locate any new publications. The following words were be used to search the websites: Cystic fibrosis, Epidemiology, Incidence, Prevalence, Middle East, MENA, North Africa, Gulf, GCC, Arab, Kuwait, Qatar, Saudi Arabia, Oman, Bahrain, United Arab Emirates, Morocco, Algeria, Libya, Tunis, Egypt, Sudan, Yemen, Jordan, Lebanon, Syria, Iraq, Palestine, Mauretania, Somalia, Djibouti, and Comoros.

\section{Inclusion and exclusion criteria}

The inclusion criteria are (1) published about one of the Arab countries (Kuwait, Qatar, Saudi Arabia, Oman, Bahrain, United Arab Emirates, Morocco, Algeria, Libya, Tunis, Egypt, Sudan, Yemen, Jordan, Lebanon, Syria, Iraq, Palestine, Mauretania, Somalia, Djibouti, Comoros); (2) reports either the prevalence or the incidence of cystic fibrosis; (3) no year, gender, or age restriction; and (4) published in one of two languages Arabic or English.

The exclusion criteria are (1) not published about one of the Arab countries (Kuwait, Qatar, Saudi Arabia, Oman, Bahrain, United Arab Emirates, Morocco, Algeria, Libya, Tunis, Egypt, Sudan, Yemen, Jordan, Lebanon, Syria, Iraq, Palestine, Mauretania, Somalia, Djibouti, Comoros), (2) not reporting either the prevalence or the incidence of cystic fibrosis, (3) published in any other language other than Arabic or English, and (4) duplicates and review papers.

\section{Screening strategy}

Two groups of independent reviewers (NO and DD, YH and CE) screened the literature through three stages. The first stage involved screening the title and abstract, the second by screening the full text, and the third by reviewing all the articles that have been included and excluded.

\section{Quality assessment}

The same two reviewer groups independently conducted the quality assessment phase based on an 8-point scoring system designed for the appraisal of studies reporting the prevalence or incidence of a health related outcome set by Loney et al. [8]. Any discrepancies were decided on by a third reviewer (SH), and any further disagreements were decided on by discussing among authors.

\section{Outcome measures}

Outcome measures were the rates of incidence and prevalence of cystic fibrosis in the populations studied.

\section{Data extraction and reporting}

Data extraction was conducted using a data extraction sheet designed for the purpose of this study. The extraction was conducted by the same two independent reviewer groups (NO and DE, YH and CE). Reporting of the data followed the Preferred Reporting Items for Systematic Reviews and Meta-Analyses (PRISMA) checklist [9]. The following variables were reported: study type, study setting, population, sample size, gender, age, prevalence, incidence, and consanguinity.

\section{Data analysis}

Due to the heterogeneous characteristics of the studies and the heterogeneity of the cohort samples used in the studies that entered this review, no data analysis was conducted. Therefore, no pooled estimates were reported.

\section{Results}

A total of 188 articles were retrieved from PubMed on February 4, 2019. Of which, 159 were excluded after screening the title and abstract. Among the remaining 29 articles, 27 full texts were retrieved, of which 13 were found to be eligible to enter the review. An additional 23 articles were found through snowballing, of which 20 were excluded. A second search was conducted on September 22, 2019, and added 16 new publications that were not present in the initial search on February 22, 2019, of which 15 were excluded. A total of 17 articles were found to be eligible and entered this systematic review. The study selection and screening process is summarized in the PRISMA flow diagram illustrated in Fig. 1. 
Fig. 1 A flow diagram detailing the search and selection process of this systematic review

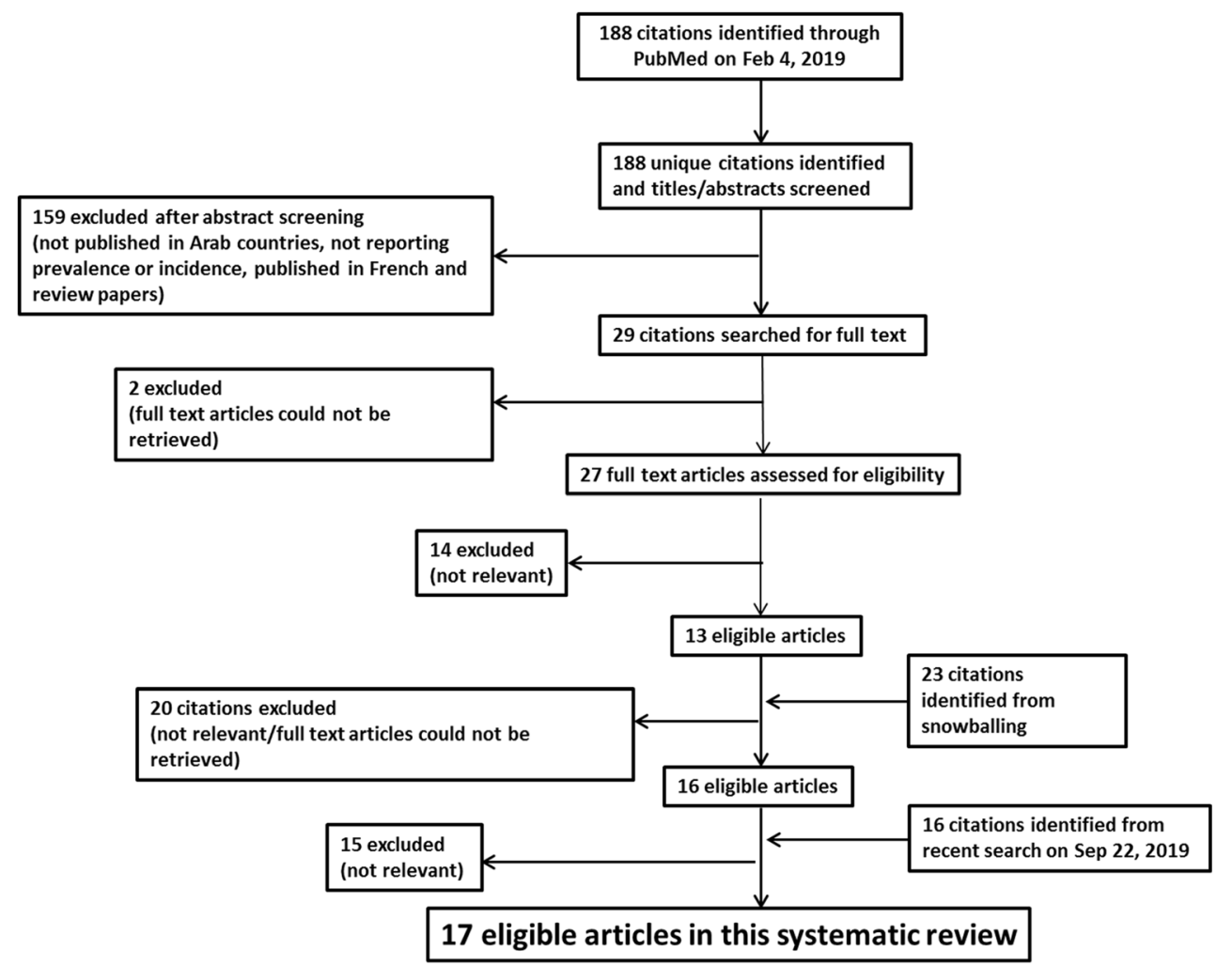

\section{Study characteristics}

Table 1 summarizes the key study characteristics of the included articles in this review. Articles that have been reviewed were published from 1986 onwards. The most recent publication was from 2018. Studies were undertaken in 9 Arab countries: Bahrain (2), Egypt (2), Jordan (2), Kuwait (1), Morocco (1), Oman (1), Saudi Arabia (5), Tunisia (1), and United Arab Emirates (2). Most of the articles were hospital series of relatively small samples. The studies employed different methodological approaches; 7 retrospective studies, 4 cross-sectional, 3 prospective, 1 pilot study, 1 retrospective/prospective, and 1 case report. The studies included mainly children and newborns. Few studies included either adults or mixed age population (children and adults). The sample size ranged from 10 to 7686 .

The table also provides a summary of the epidemiological indicators of CF in each of the 9 countries. The epidemiology of the disease was described using incidence, birth incidence, prevalence range, point prevalence (cases per 100,000), or percentage (i.e., frequency of people suspected of having the disease). Five of the 9 countries reported both incidence and prevalence figures, while studies from Saudi Arabia and Jordan reported incidence figures only, and those form Morocco and the United Arab Emirates (UAE) had reported on the prevalence alone. Given that establishing a true prevalence of a rare disease is particularly challenging [30], the estimated prevalence was derived from the incidence and extrapolated to the population, or predicted from the frequency of CF mutated alleles under the condition of consanguinity.
The incidence and prevalence reported in the table from Egypt $[12,14]$ were obtained from another article by AbdelSalam et al. [13], that we were not able to retrieve, so the figures were retained and the other study was referenced. As for Saudi Arabia, the incidence was obtained from another article by Mathew et al. [20] and reported in the other 4 articles from Saudi Arabia. In Jordan, the incidence was obtained from Nazer [15] and reported in the second article from Jordan [16].

\section{Quality assessment}

The quality of each study was assessed against an eight criteria tool developed by Loney et al. [8] and designed to critically appraise epidemiological health research literature. The tool was comprised of items that assess the following components: study design and sampling methods, sampling frame, sample size, appropriate measurement, unbiased measurement, response rate, results, and study subjects. Each criterion weighs 1 point, making 8 the maximum score possible [8].

We accommodated a minor modification by assigning half a point $(0.5)$ when criteria were "partially met". The studies that have been reviewed scored an overall quality score ranging from 1.5 to 6.5 . Articles generally scored well in areas of measures were the standards, and detailed description of the setting and study subjects. The main limitations were not implementing an appropriate study design and sampling method, inadequate sample size, and not adequately 


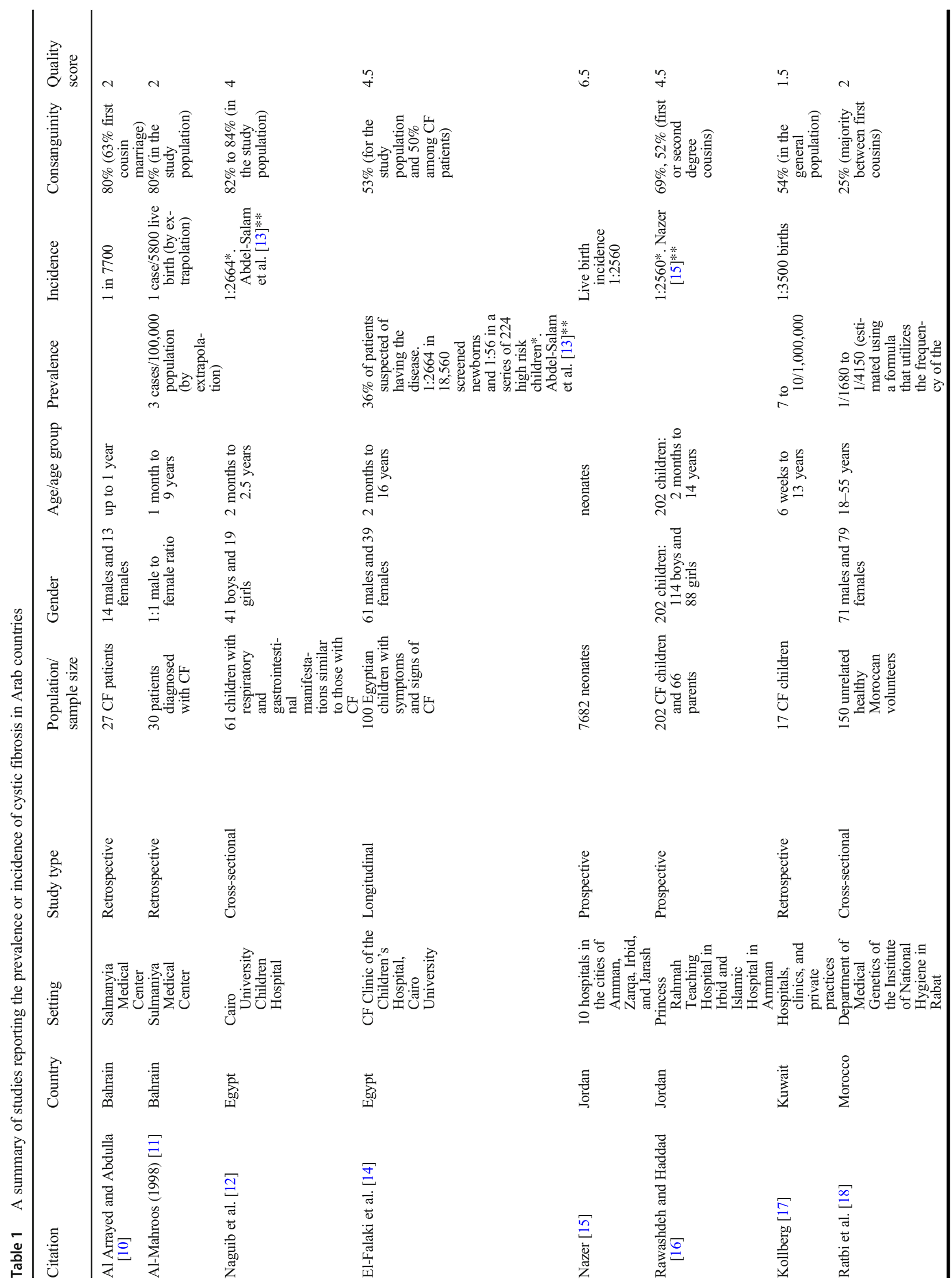




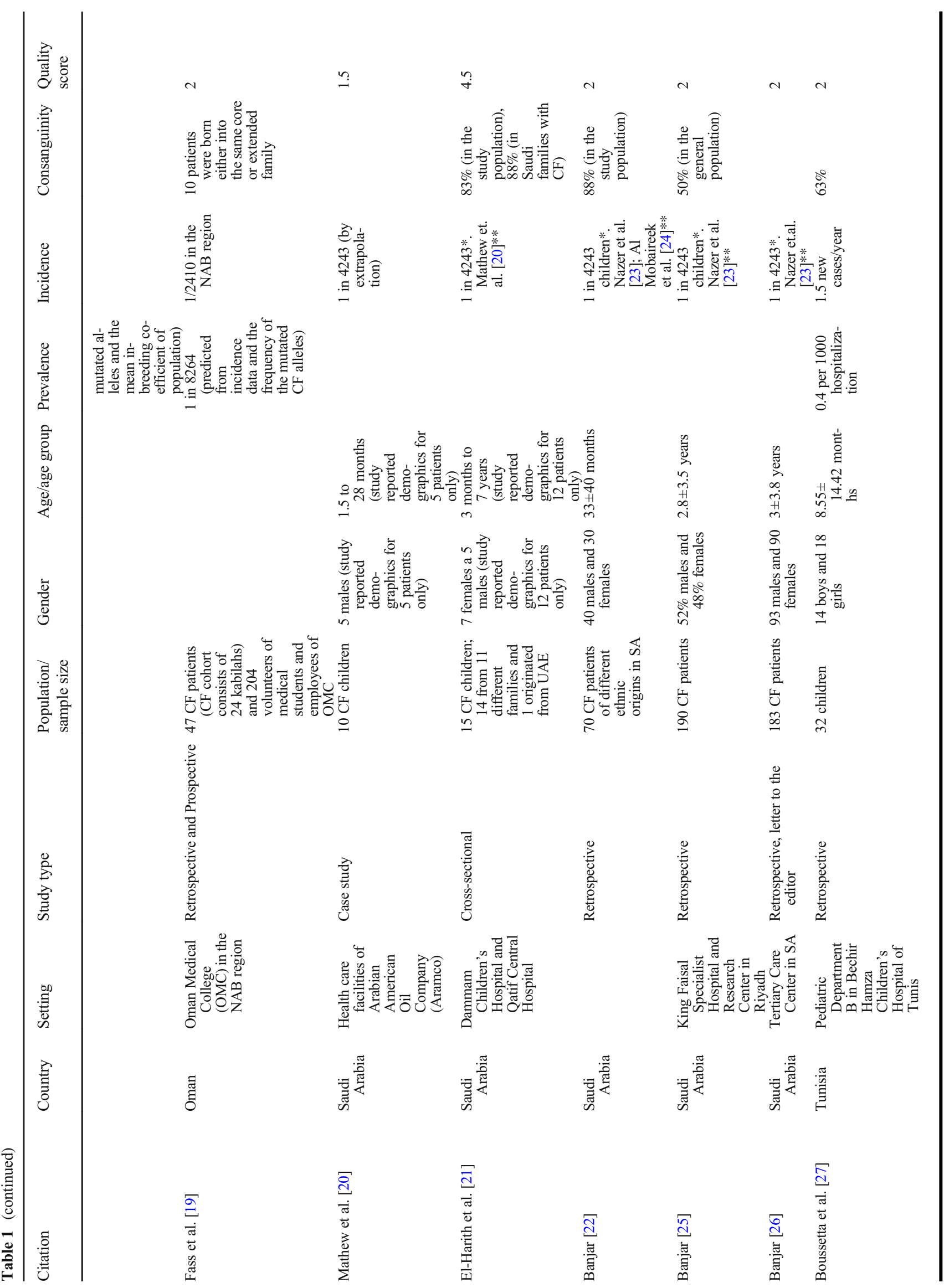


interpreting the results in terms of reporting the confidence interval and sub-group analysis. These limitations are most likely due to the nature of the disease and the studies as well. Yet, the low total score of a reviewed study does not infer low validity as the rarity of the disease makes it infeasible to follow the guidelines formalized for ideal epidemiological studies of a common health problem.

\section{CF incidence and prevalence by country}

Bahrain Two retrospective studies were conducted in Bahrain by Al Arrayed and Abdulla [10], and Al-Mahroos [11]. All CF cases from 1978 to 1995 were identified. Based on extrapolations made from each study to the population, Al Arrayed and Abdulla estimated the incidence of CF to be 1 in 7700 [10], whereas, Al-Mahroos reported an incidence of 1 case per 5800 Bahraini live births, and a prevalence of 3 cases per 100,000 population [11].

Egypt Two groups of authors conducted 2 studies to determine the presence of $\mathrm{CF}$ among referred patients with clinical manifestations similar to those reported in CF patients. Naguib et al. reported a prevalence rate of 1:2664 [12]. Whereas, ElFalaki et al. reported a prevalence rate of 1:2664 in 18,560 screened newborns and 1:56 in a serious of 224 high risk children [14]. The outcomes of El-Falaki et al. study showed a high frequency of CF; $36 \%$ of patients referred to the CF Clinic of Children's Hospital in Cairo and who are suspected clinically of having the disease, do in fact have the disease. The figures reported in both studies by Naguib et al. and ElFalaki et al. were based on the original epidemiological study by Abdel-Salam et al. who screened 18,650 Egyptian neonates for CF [13]; however, this article could not be retrieved.

Jordan The incidence of CF was found to be 1:2560 live births in a prospective study that screened 7682 neonates from different hospitals in Jordan [15]. The same rate was also reported in a second study by Rawashdeh and Haddad who also reported that 202 cases diagnosed in Jordan with CF over the period from 1990 to 1999 , and concluded that this number of CF patients points to a high prevalence of CF in Jordan [16].

Kuwait In 1986, Kollberg conducted a retrospective study to estimate the prevalence of CF in Kuwait. Due to incomplete medical records and lack of registries, most of the figures were obtained via personal communication with practitioners. Over the period from 1980 to 1985,17 patients have been diagnosed with CF. The clinical presentations of these patients were described by Kollberg, who estimated the prevalence of CF among Kuwaiti and other Arab population in Kuwait to be 7 to 10 per $1,000,000$. Building on figures from this study and comparing to other Caucasian countries, the minimum incidence in Kuwait was estimated to be 1:3500 births [17]. 
Morocco Ratbi et al. [18] conducted a prospective study to estimate the prevalence of CF mutations in Morocco. During a blood donation program in Rabat, blood samples were collected from 150 unrelated health Moroccan volunteers. The samples were screened for 32 CFTR mutations. The frequency of the mutated allele was calculated considering $25 \%$ consanguinity rate. Using a formula that utilizes the frequency of the mutation and the mean inbreeding coefficient of population, an estimate of the expected prevalence was obtained. The authors estimated that the prevalence of CF in Morocco would range from $1 / 1680$ to $1 / 4150$. However, this figure may be overestimated because of the effect of the sample size. Therefore, they suggested that CF prevalence in Morocco is similar to rates that are found in Europe [18].

Oman The study conducted by Fass et al. [19] included both retrospective and prospective analyses to estimate $\mathrm{CF}$ prevalence in the NAB region and in Oman as well. Retrospectively, 47 patients with $\mathrm{CF}$ were identified during the period of 1998 to 2012 in the NAB region. On the other hand, a total of 204 unrelated Omani volunteers from Oman Medical Collage of Sohar in the NAB region were recruited for survey and screening for CF-causing mutations. Based on the number of $\mathrm{CF}$ cases identified over the 14-year period and the average annual rate birth, the incidence was estimated to be $1 / 2410$ affected newborns in the NAB region, whereas the prevalence of the disease was estimated based on the genotype frequency resulting in a predicted prevalence of 1/8264 was reported for Oman [19].

Saudi Arabia Five of the reviewed articles were from Saudi Arabia. The earliest was a case study done by Mathew et al. [20]. The authors reported 10 cases with CF in the health care facilities of the Eastern province of Saudi Arabia. They particularly presented the cases of two siblings with $\mathrm{CF}$. Based on extrapolations made from the reported cases to the population, the incidence was estimated to be 1 in 4243 among Saudi Arabian children [20]. The same figure was reported in the other four studies from Saudi Arabia, El-Harith et.al. [21], Banjar [22], Banjar [25], and Banjar [26].

Tunisia Boussetta et al. [27] conducted a retrospective study covering the period of 1996 to 2016 in order to include all CF patients from the Pediatric Department B of The Children's Hospital of Tunis. The sample included 32 children (14 boys and 18 girls) diagnosed with $\mathrm{CF}$. The diagnosis of $\mathrm{CF}$ was confirmed by carrying out 2 sweat tests coupled with mutational analysis. The incidence and prevalence were estimated as 1.5 new cases/year and 0.4/1000 hospitalizations, respectively [27].

United Arab Emirates (UAE) Two articles were reviewed from the UAE, the first by Frossard et al. which aimed at characterizing $\mathrm{CF}$ alleles among UAE nationals. Based on the frequency of $\mathrm{CF}$ mutations in $23 \mathrm{CF}$ cases, the authors estimated a conservative $\mathrm{CF}$ prevalence of $1 / 15,000$ [28]. In 1999, Frossard et al. designed a pilot study aimed at screening CFTR genes in a random sample of 400 unrelated UAE nationals, with a view to estimate the prevalence of $\mathrm{CF}$ in the country. The authors reported a similar estimated figure of $\mathrm{CF}$ prevalence (1:15876) among the Emirati population [29].

\section{Discussion}

This review provides a foundation for future researchers investigating the various aspects of cystic fibrosis in Arab countries, as it reports on the epidemiological components of the disease. Previous work in this area involved a recent review which covered the spectrum of mutations for cystic fibrosis in Arab countries, and briefly commented on the epidemiological aspects of the disease [31]. Another article reported estimated incidence rates from 13 Middle Eastern countries [32].

In this systematic review and based on the literature reviewed here, it is worth highlighting a few points; first, in relation to estimates like the prevalence and incidence in Arab countries, these have been reported in various ways and calculated through various methods. Studies have attempted to calculate these figures based on visitors to one center or on a sample size for one study, or predicted it based on screening results for a certain population. Second, in regard to the study samples, a large variety or heterogeneity of the samples used in the studies was observed. For an example, some studies used a population from a certain region of a certain country. This lack of standardization disallowed the calculation of any pooled estimates.

Generally speaking, there is a methodological challenge that is specific to measuring small populations [33]. The reported incidence/prevalence in the reviewed articles is an estimate derived from a particular sample, in some cases based on a small number of participants. The prevalence derived from incidence may overestimate the diagnosed prevalence [30], as reported by Ratbi et al. [18]. On the other hand, the incidence might be underestimated due to lack of ascertainment. Many patients with CF are thought to be hidden among the cohort of recurrent respiratory and gastrointestinal infections due to lack of efficient diagnostic facilities, and therefore, are missed out [17]. Another study reported the problem of missing out on isolated affected families living in remote desert areas (Frossard et al. 1999).

A third point involves the lack of national $\mathrm{CF}$ registries, if any, in Arab countries, along with the lack of the longitudinal data coming from it, has reflected on the lack of proper estimates of these patients, and not to mention the lack of demographics and other information that would have been useful in monitoring the health of these patients, as in clinical data or those related to quality of life or other aspects of the disease. 
A fourth point involves the age of the studies, as a large segment of the studies reviewed were $2-3$ decades old. Given that the incidence of a rare disease may increase over time with improved diagnostic definitions, technologies, intensified treatment, and expertise adopted into widespread clinical practice [30]; thus, studies aiming to determine incidence or prevalence or both should be revisited with emphasis on large scale population-based studies, especially, in those countries with no reports on the epidemiological aspects of the disease.

Collectively, these points have obviously reflected on the scores for the quality assessment of the studies, which was presented through the lack of several components in this assessment, which is also due to the fact that some studies were not original research but rather reported figures from other older studies done by other authors. Among the limitations of this study is that it was restricted to one database alone.

In all, there is an obvious need for further research related to $\mathrm{CF}$ in Arab countries, with emphasis on longitudinal studies to aid in further understanding CF-related complications, quality of life, and life expectation. CF registries, on the local and regional level, are necessary as well in order to ascertain the true number of CF patients across Arab countries. This would aid in monitoring various aspects related to population health, allow for the conduction of longitudinal studies, and more importantly will allow for comparisons between registries [2].

\section{Conclusion}

The establishment of CF registries is definitely a priority in the region, as well as recognizing the need for more research involving CF patients particularly those involving the epidemiological and clinical characteristics of these patients, as identifying this information will supplement current global cystic fibrosis figures, which in turn aids in supplementing future global guidelines, awareness efforts, and research activities.

Acknowledgements The publication of this article was funded by the Qatar National Library. Contact details: openaccess@qnl.qa

Author contributions $\mathrm{YH}, \mathrm{NO}, \mathrm{DE}$, and $\mathrm{CE}$ reviewed the literature, conducted the quality assessment, and extracted the data. NO drafted the manuscript. AP conducted the search on PubMed. PC was involved in data analysis and statistical advice. SH conceptualized the study, developed the methods, and drafted the manuscript. WG was the project manager. IJ was the senior author and advisor on the project. All authors read and approved the final manuscript.

Funding Open access funding provided by the Qatar National Library.

Data availability None.

\section{Declarations}

Ethics approval and consent to participate Not applicable.
Consent for publication Not applicable.

\section{Competing interests None.}

Open Access This article is licensed under a Creative Commons Attribution 4.0 International License, which permits use, sharing, adaptation, distribution and reproduction in any medium or format, as long as you give appropriate credit to the original author(s) and the source, provide a link to the Creative Commons licence, and indicate if changes were made. The images or other third party material in this article are included in the article's Creative Commons licence, unless indicated otherwise in a credit line to the material. If material is not included in the article's Creative Commons licence and your intended use is not permitted by statutory regulation or exceeds the permitted use, you will need to obtain permission directly from the copyright holder. To view a copy of this licence, visit http://creativecommons.org/licenses/by/4.0/.

\section{References}

1. Elborn JS. Cystic fibrosis. Lancet. 2016;388(10059):2519-31.

2. Jackson A, Goss C. Epidemiology of CF: how registries can be used to advance our understanding of the CF population. J Cyst Fibros. 2018;17(3):297-305.

3. Antoniou S, Elston C. Cystic fibrosis. Medicine. 2016;44(5):321-5.

4. Banjar H, Angyalosi G. The road for survival improvement of cystic fibrosis patients in Arab countries. Int J Pediatr Adolesc Med. 2015;2(2):47-58.

5. O'Sullivan BP, Freedman SD. Cystic fibrosis. Lancet. 2009;373(9678):1891-904.

6. Thursfield RM, Davies JC. Cystic fibrosis: therapies targeting specific gene defects. Paediatr Respir Rev. 2012;13(4):215-9.

7. Wohlin, C., Guidelines for snowballing in systematic literature studies and a replication in software engineering, in Proceedings of the 18th International Conference on Evaluation and Assessment in Software Engineering. 2014, ACM: London, England, United Kingdom. p. 1-10.

8. Loney, P., et al., Critical appraisal of the health research literature: prevalence or incidence of a health problem. Vol. 19. 1998. 170-6.

9. Moher D, Liberati A, Tetzlaff J, Altman DG, The PRISMA Group. Preferred reporting items for systematic reviews and meta-analyses: the PRISMA statement. PLoS Med. 2009;6(7):e1000097.

10. Arrayed SSA, Abdulla F. Incidence of cystic fibrosis in Bahrain. J Bahrain Med Soc. 1996;8(3).

11. Al-Mahroos F. Cystic fibrosis in Bahrain incidence, phenotype, and outcome. J Trop Pediatr. 1998;44(1):35-9.

12. Naguib ML, Schrijver I, Gardner P, Pique LM, Doss SS, Abu Zekry MA, et al. Cystic fibrosis detection in high-risk Egyptian children and CFTR mutation analysis. J Cyst Fibros. 2007;6(2):111-6.

13. Abdel-Salam ESS, Awad M, El-Marsafy A, Abdel-Meguid IE, Azmy J. Cystic fibrosis in Egyptian children: neonatal screening and high risk groups. JAC. 1993;4:313-7.

14. El-Falaki MM, et al. Profile of cystic fibrosis in a single referral center in Egypt. J Adv Res. 2014;5(5):563-8.

15. Nazer HM. Early diagnosis of cystic fibrosis in Jordanian children. J Trop Pediatr. 1992;38(3):113-5.

16. Rawashdeh M, Manal H. Cystic fibrosis in Arabs: a prototype from Jordan. Ann Trop Paediatr. 2000;20(4):283-6.

17. Kollberg H. Cystic fibrosis in Kuwait. J Trop Pediatr. 1986;32(6): 293-4.

18. Ratbi I, Génin E, Legendre M, le Floch A, Costa C, CherkaouiDeqqaqi $\mathrm{S}$, et al. Cystic fibrosis carrier frequency and estimated 
prevalence of the disease in Morocco. J Cyst Fibros. 2008;7(5): 440-3.

19. Fass UW, al-Salmani M, Bendahhou S, Shivalingam G, Norrish C, Hebal K, et al. Defining a mutational panel and predicting the prevalence of cystic fibrosis in Oman. Sultan Qaboos Univ Med J. 2014;14(3):e323-9.

20. Mathew PM, Hamdan JA, Nazer H. Cystic fibrosis presenting with recurrent vomiting and metabolic alkalosis. Eur J Pediatr. 1991;150(4):264-6.

21. El-Harith EA, et al. Novel and characteristic CFTR mutations in Saudi Arab children with severe cystic fibrosis. J Med Genet. 1997;34(12):996-9.

22. Banjar H. Geographic distribution of cystic fibrosis transmembrane regulator gene mutations in Saudi Arabia. East Mediterr Health J. 1999;5(6):1230-5.

23. Nazer H, Riff E, Sakati N, Mathew R, Majeed-Saidan MA, Harfi H. Cystic fibrosis in Saudi Arabia. European Journal of Pediatrics. 1989;148: 330-2.

24. Al-Mobaireek KF, Abdullah A. Cystic fibrosis in Saudi Arabia: common and rare presentations. Annals of Tropical Paediatrics. 1995;15(4):269-72.

25. Banjar H. Morbidity and mortality data of cystic fibrosis patients. Saudi Med J. 2003;24(7):730-5.

26. Banjar H. Mortality data for cystic fibrosis patients in a tertiary care center in Saudi Arabia [1]. Ann Saudi Med. 2003;23(6):416-7.
27. Boussetta K, Khalsi F, Bahri Y, Belhadj I, Tinsa F, Messaoud TB, et al. Cystic fibrosis in Tunisian children: a review of 32 children. Afr Health Sci. 2018;18(3):664-70.

28. Frossard PM, Girodon E, Dawson KP, Ghanem N, Plassa F, Lestringant $\mathrm{GG}$, et al. Identification of cystic fibrosis mutations in the United Arab Emirates. Mutations in brief no. 133. Online. Hum Mutat. 1998;11(5):412-3.

29. Frossard PM, Lestringant G, Girodon E, Goossens M, Dawson KP. Determination of the prevalence of cystic fibrosis in the United Arab Emirates by genetic carrier screening. Clin Genet. 1999;55(6):496-7.

30. Auvin S, Irwin J, Abi-Aad P, Battersby A. The problem of rarity: estimation of prevalence in rare disease. Value Health. 2018;21(5): $501-7$.

31. Al-Sadeq D, et al. Spectrum of mutations of cystic fibrosis in the 22 Arab countries: a systematic review. Respirology. 2018;24(2):12736.

32. Hider AM. Letter to the editor: cystic fibrosis in the Middle East: an awareness analysis. J Cyst Fibros. 2019;18(4):e40-1.

33. Rahme E, Joseph L. Estimating the prevalence of a rare disease: adjusted maximum likelihood. J R Stat Soc Ser D (The Statistician). 1998;47(1):149-58.

Publisher's Note Springer Nature remains neutral with regard to jurisdictional claims in published maps and institutional affiliations. 